\title{
Mucus Flow in Human Lung Airways: Effects of Air Velocity, Cilia Tip Velocity and Porosity Parameter
}

\author{
S. M. Tripathee ${ }^{1}$, V.S. Verma ${ }^{2}$ \\ ${ }^{1}$ BabaSaheb Bhim Rao Ambedkar University, Lucknow \\ ${ }^{2}$ DDU Gorakhpur University, Gorakhpur
}

\begin{abstract}
In this paper, a two layer steady state mathematical model is presented to study the mucus flow in the human lung airways airflow, cilia beating and porosity parameter due to formation of ciliary porous bed in contact with the epithelium.The effect of airflow is considered by prescribing the air-velocity at the mucus-air interface. The effects of pressure gradients present in the fluid and gravitational force are also taken into account. The serous layer fluid and mucus both are taken as Newtonian fluid. It has been shown that mucus flow rate decreases as the viscosities of mucus and serous layer fluid increase and it increases as the air-velocity at the mucus-air interface, pressure drop and gravitational force increase. It has been also observed that mucus flow rate increases as the porosity parameter increase
\end{abstract}

Keywords: Mucus flow, human lung, cilia beating.

\section{Introduction}

Mucus flow in the lung has been studied by several investigators. In recent decades, various models are presented and analysed. Agarwal and Verma (1997) studied the mucus transport in the respiratory tracts and explained the effects of various parameters on mucus transport including the effects of air-motion and porosity parameter.

In view of above, in this chapter, a two-layer circular steady state mathematical model is presented to study the mucus flow in the human lung airways by taking into account the effects of cilia beating as well as porosity parameter incorporated due to certain immotile cilia forming porous matrix bed in the serous sub layer in contact with the epithelium. The effect of air-velocity due to air motion at the mucus-air interface is also taken in the model. The effects of pressure gradient and gravitational force are also taken into consideration.

\section{Mathematical Model}

In real situation, the airways in the human lung are circular in nature. Therefore, the physical situation of movement in the human lung is idealized by circular tube geometry, the inner surface wall being ciliated (Fig 1) and it is assumed that the central lumen is filled by air surrounded by mucus (a highly viscous fluid), which is covered by a serous layer fluid which is divided in two sub-layers, one in contact with epithelium and other in contact with mucus where flow may occur due to pressure gradient as considered by Beavers and Joseph (1967).

It is assumed that certain cilia beat continuously and certain cilia are immotile which form a porous bed in contact with the epithelium, where no net flow is considered. The effect of air flow is incorporated by prescribing the air -velocity at the mucus-air interface as a boundary condition.
The equations governing the flow of mucus and serous layer fluid under steady state and low Reynolds number flow approximations by taking the effect of gravitational force into account in the direction of flow, are written as follows:

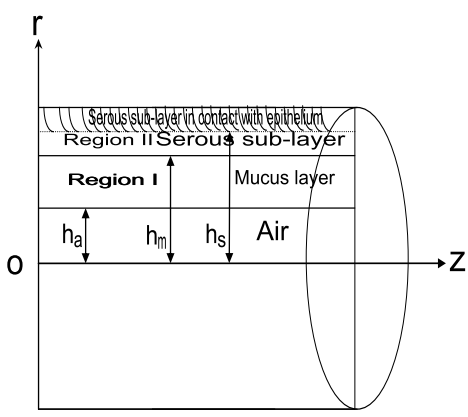

Figure 1: A circular model for mucus flow in lung airways

Region-I: Mucus layer $\left(\boldsymbol{h}_{\boldsymbol{a}} \leq \boldsymbol{r} \leq \boldsymbol{h}_{\boldsymbol{m}}\right)$ :

$$
\frac{\mu_{m}}{r} \frac{\partial}{\partial r}\left(r \frac{\partial u_{m}}{\partial r}\right)=\frac{\partial p}{\partial z}-\rho_{m} g \cos \alpha
$$

Rigion-II: Serous layer $\left(\boldsymbol{h}_{\boldsymbol{m}} \leq \boldsymbol{r} \leq \boldsymbol{h}_{\boldsymbol{s}}\right)$ :

$$
\frac{\mu_{s}}{r} \frac{\partial}{\partial r}\left(r \frac{\partial u_{s}}{\partial r}\right)=\frac{\partial p}{\partial z}-\rho_{s} g \cos \alpha
$$

where $p$ is the pressure which is constant across the layer, $u_{m}$ and $u_{s}$ are the velocity component of mucus and serous layer fluid respectively in $z$-direction; $\rho_{m}, \mu_{m}$ and $\rho_{s}, \mu_{s}$ are their respective densities and viscosities; $g$ is the acceleration due to gravity; $\alpha$ is the angle by which the airway under consideration is inclined with the vertical; $h_{a}$, $h_{m}$ and $h_{s}$ are the thickness measured from the axis $o z$ to the mucus air interface, serous-mucus interface and interface between the two serous sub-layers respectively as shown in the Fig. 1 .

$$
\begin{aligned}
& \text { Boundary Conditions: } \\
& \qquad \begin{array}{l}
u_{m}=U_{a}, r=h_{a} \\
u_{s}=U_{0}+\beta \frac{\partial u_{s}}{\partial r}, r=h_{s}
\end{array}
\end{aligned}
$$




\section{International Journal of Science and Research (IJSR) \\ ISSN (Online): 2319-7064 \\ Index Copernicus Value (2013): 6.14 | Impact Factor (2014): 5.611}

where $U_{a}$ is the prescribed air-velocity at the mucus-air interface which incorporates the effect of airflow, $U_{0}$ is the mean velocity of cilia tips while beating and $\beta$ is the porosity parameter whose dimension is same as that of fluid layer thickness. Condition (6.3) incorporates the effect of air-motion and condition (6.4) incorporates the effect of cilia beating and porous matrix bed formed by certain immotile cilia.

\section{Matching Conditions:}

$$
\begin{aligned}
& u_{m}=u_{s}=U_{1}, r=h_{m} \\
& \mu_{m} \frac{\partial u_{m}}{\partial r}=\mu_{s} \frac{\partial u_{s}}{\partial r}, r=h_{m}
\end{aligned}
$$

where $U_{1}$ is the mucus-serous layer interface velocity to be determined by using condition (6.6). The conditions (5) and (6) imply that the velocities and shear stresses are continuous at the mucus-serous sub-layer interface.

\section{Analytical Solution}

Solving equations (6.1) and (6.2) and using boundary and matching conditions (6.3)-(6.6), we get

$$
\begin{aligned}
& u_{m}=U_{a}\left[1-\frac{\ln \left(\frac{r}{h_{a}}\right)}{\ln \left(\frac{h_{m}}{h_{a}}\right)}\right]+U_{1}\left[\frac{\ln \left(\frac{r}{h_{a}}\right)}{\ln \left(\frac{h_{m}}{h_{a}}\right)}\right]+\frac{\phi_{m}}{4 \mu_{m}}\left[\left(r^{2}-h_{a}^{2}\right)-\right. \\
& \text { hm2-ha2ln rhalnhmha (7) }
\end{aligned}
$$

And

$$
u_{s}=\frac{U_{1}\left\{\frac{\beta}{h_{s}}+\ln \left(\frac{r}{h_{s}}\right)\right\}}{\left\{\frac{\beta}{h_{s}}+\ln \left(\frac{h_{m}}{h_{s}}\right)\right\}}-\frac{U_{0} \ln \left(\frac{r}{h_{m}}\right)}{\left\{\frac{\beta}{h_{s}}+\ln \left(\frac{h_{m}}{h_{s}}\right)\right\}}+\frac{\phi_{s}}{4 \mu_{s}}\left[\left(r^{2}-h_{m}^{2}\right)-\right.
$$

$h m 2-h s 2+2 \beta h s\{\beta h s+\ln h m h s\} \ln r h m$

where $U_{1}$ is given by the following relation:

$$
\begin{aligned}
& U_{1}\left[\frac{\mu_{s} \ln \left(\frac{h_{m}}{h_{a}}\right)-\mu_{m}\left\{\frac{\beta}{h_{S}}+\ln \left(\frac{h_{m}}{h_{s}}\right)\right\}}{\ln \left(\frac{h_{m}}{h_{a}}\right)\left\{\frac{\beta}{h_{s}}+\ln \left(\frac{h_{m}}{h_{s}}\right)\right\}}\right]=\frac{\phi_{s}}{4}\left[\frac{\left(h_{m}^{2}-h_{S}^{2}+2 \beta h_{s}\right)}{\left\{\frac{\beta}{h_{s}}+\ln \left(\frac{h_{m}}{h_{s}}\right)\right\}}-2 h_{m}^{2}+\right. \\
& \frac{\phi_{m}}{4}\left[2 h_{m}^{2}-\frac{\left(h_{m}^{2}-h_{a}^{2}\right)}{\ln \left(\frac{h_{m}}{h_{a}}\right)}\right]-\frac{\mu_{m} U_{a}}{\ln \left(\frac{h_{m}}{h_{a}}\right)}+\frac{\mu_{s} U_{0}}{\left\{\frac{\beta}{h_{s}}+\ln \left(\frac{h_{m}}{h_{s}}\right)\right\}} \text { (9) }
\end{aligned}
$$

and $\phi_{m}=\frac{\partial p}{\partial z}-\rho_{m} g \cos \alpha, \phi_{s}=\frac{\partial p}{\partial z}-\rho_{s} g \cos \alpha(\mathbf{1 0})$

Now, the volumetric flow rate in two layers i.e. $Q_{m}$ and $Q_{s}$ are given by :

$Q_{m}=\int_{h_{a}}^{h_{m}} 2 \pi r u_{s} d r$ and $Q_{s}=\int_{h_{m}}^{h_{s}} 2 \pi r u_{s} d r$

which after using (7) and (8) are obtained as follows:

$$
\begin{aligned}
Q_{m}=\frac{\pi U_{a}}{2}\left[\frac{\left(h_{m}^{2}-h_{a}^{2}\right)}{\ln \left(\frac{h_{m}}{h_{a}}\right)}-2 h_{a}^{2}\right]-\frac{\pi U_{1}}{2}\left[\frac{\left(h_{m}^{2}-h_{a}^{2}\right)}{\ln \left(\frac{h_{m}}{h_{a}}\right)}-2 h_{m}^{2}\right] \\
-\frac{\pi \phi_{m}}{8 \mu_{m}}\left[\left(h_{m}^{4}-h_{a}^{4}\right)-\frac{\left(h_{m}^{2}-h_{a}^{2}\right)^{2}}{\ln \left(\frac{h_{m}}{h_{a}}\right)}\right]
\end{aligned}
$$

and

$$
\begin{aligned}
& Q_{s}=\frac{\pi U_{1}}{2}\left[\frac{2 \beta\left(h_{s}^{2}-h_{m}^{2}\right)-h_{s}\left\{\left(h_{s}^{2}-h_{m}^{2}\right)+2 h_{m}^{2} \ln \left(\frac{h_{m}}{h_{s}}\right)\right\}}{h_{s}\left\{\frac{\beta}{h_{s}}+\ln \left(\frac{h_{m}}{h_{s}}\right)\right\}}\right]+ \\
& \frac{\pi h_{s} U_{0}}{2}\left[\frac{\left.\left(h_{s}^{2}-h_{m}^{2}\right)+2 h_{s}^{2} \ln \left(\frac{h_{m}}{h_{s}}\right)\right)}{h_{s}\left\{\frac{\beta}{h_{s}}+\ln \left(\frac{h_{m}}{h_{s}}\right)\right\}}\right] \\
& -\frac{\pi \phi_{s}}{8 \mu_{s}}\left[\frac{\left(h_{s}^{2}-h_{m}^{2}-2 \beta h_{s}\right)\left(h_{s}^{2}-h_{m}^{2}+2 h_{s}^{2} \ln \left(\frac{h_{m}}{h_{s}}\right)\right)}{\left\{\frac{\beta}{h_{s}}+\ln \left(\frac{h_{m}}{h_{s}}\right)\right\}}-\left(h_{s}^{2}-h_{m}^{2}\right)^{2}\right]
\end{aligned}
$$

where $U_{1}$ is given by

$$
\begin{aligned}
& U_{1}=-\frac{\phi_{m}}{4 \mu_{m}}\left[2 h_{m}^{2} \ln \left(\frac{h_{m}}{h_{a}}\right)-\left(h_{m}^{2}-h_{a}^{2}\right)\right](1+A) \\
& -\frac{\phi_{s}}{4 \mu_{m}}\left[\frac{\left\{2 \beta h_{s}-\left(h_{s}^{2}-h_{m}^{2}\right)\right\}}{\left\{\frac{\beta}{h_{s}}+\ln \left(\frac{h_{m}}{h_{s}}\right)\right\}}-2 h_{m}^{2}\right] \ln \left(\frac{h_{m}}{h_{a}}\right)(1+A) \\
& \text { where } A=\left(\frac{\mu_{s}}{\mu_{m}}\right)\left[\frac{\ln \left(\frac{h_{m}}{h_{a}}\right)}{\left\{\frac{\beta}{h_{s}}+\ln \left(\frac{h_{m}}{h_{s}}\right)\right\}}\right]
\end{aligned}
$$

It can be seen by using the equation of fluid continuity that $Q_{m}$ and $Q_{s}$ are constant, therefore, from equations (11) and (12), we note that $-\frac{\partial p}{\partial z}$ is also constant. Hence, replacing it by pressure drop over the length $L$ of cilia forming porous bed zone, the expressions for the flow rate can be written as:

$$
\begin{aligned}
Q_{m}=\frac{\pi \phi_{m_{0}}}{8 \mu_{m}} & {\left[\left(h_{m}^{4}-h_{a}^{4}\right)-\frac{\left(h_{m}^{2}-h_{a}^{2}\right)^{2}}{\ln \left(\frac{h_{m}}{h_{a}}\right)}\right] } \\
& +\frac{\pi U_{a}}{2}\left[\frac{\left(h_{m}^{2}-h_{a}^{2}\right)}{\ln \left(\frac{h_{m}}{h_{a}}\right)}-2 h_{a}^{2}\right]-\frac{\pi U_{1}}{2}\left[\frac{\left(h_{m}^{2}-h_{a}^{2}\right)}{\ln \left(\frac{h_{m}}{h_{a}}\right)}-2 h_{m}^{2}\right]
\end{aligned}
$$

and

$$
\begin{aligned}
& Q_{s}=\frac{\pi U_{1}}{2}\left[\frac{2 \beta\left(h_{s}^{2}-h_{m}^{2}\right)-h_{s}\left\{\left(h_{s}^{2}-h_{m}^{2}\right)+2 h_{m}^{2} \ln \left(\frac{h_{m}}{h_{s}}\right)\right\}}{h_{s}\left\{\frac{\beta}{h_{s}}+\ln \left(\frac{h_{m}}{h_{s}}\right)\right\}}\right]+ \\
& \frac{\pi h_{s} U_{0}}{2}\left[\frac{\left.\left(h_{s}^{2}-h_{m}^{2}\right)+2 h_{s}^{2} \ln \left(\frac{h_{m}}{h_{s}}\right)\right)}{h_{s}\left\{\frac{\beta}{h_{s}}+\ln \left(\frac{h_{m}}{h_{s}}\right)\right\}}\right] \\
& +\frac{\pi \phi_{s_{0}}}{8 \mu_{s}}\left[\frac{\left(h_{s}^{2}-h_{m}^{2}-2 \beta h_{s}\right)\left\{h_{s}^{2}-h_{m}^{2}+2 h_{s}^{2} \ln \left(\frac{h_{m}}{h_{s}}\right)\right\}}{\left\{\frac{\beta}{h_{s}}+\ln \left(\frac{h_{m}}{h_{s}}\right)\right\}}-\left(h_{s}^{2}-h_{m}^{2}\right)^{2}\right]
\end{aligned}
$$

where $U_{1}$ is given by

$$
\begin{array}{r}
U_{1}=\frac{\phi_{m_{0}}}{4 \mu_{m}}\left[2 h_{m}^{2} \ln \left(\frac{h_{m}}{h_{a}}\right)-\left(h_{m}^{2}-h_{a}^{2}\right)\right](1+A) \\
+\frac{\phi_{s_{0}}}{4 \mu_{m}}\left[\frac{\left\{2 \beta h_{s}-\left(h_{s}^{2}-h_{m}^{2}\right)\right\}}{\left\{\frac{\beta}{h_{s}}+\ln \left(\frac{h_{m}}{h_{s}}\right)\right\}}-2 h_{m}^{2}\right] \ln \left(\frac{h_{m}}{h_{a}}\right)(1+A)+U_{a}(1+ \\
A)-U_{0} A(1+A)(\mathbf{1 6})
\end{array}
$$

and $\phi_{m_{0}}=\frac{\Delta p}{L}-\rho_{m} g \cos \alpha, \phi_{s_{0}}=\frac{\Delta p}{L}-\rho_{s} g \cos \alpha$ (17) where $\Delta p=p_{0}-p_{L} ; p=p_{0}$ at $z=0 ; p=p_{L}$ at $z=L$.

Now, substituting the value of $U_{1}$ from (16) in equations (14) and (15), the expressions for the volumetric flow rate may be written as:

$Q_{m}=\frac{\pi \phi_{m_{0}}}{8 \mu_{m} \ln \left(\frac{h_{m}}{h_{a}}\right)}\left[\left\{\left(h_{m}^{4}-h_{a}^{4}\right) \ln \left(\frac{h_{m}}{h_{a}}\right)-\left(h_{m}^{2}-h_{a}^{2}\right)^{2}\right\}+\right.$

$2 h m 21 n h m h a-h m 2-h a 22(1+A)+\pi \phi s 08 \mu m\{2 \beta h s-(h s 2$ -hm2) $\{\beta h s+\ln h m h s\}-2 h m 2\{2 h m 2 \ln h m h a-(h m 2-h a$

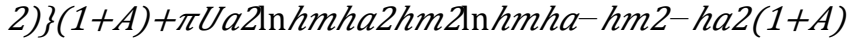
$+\quad h m 2-h a 2-2 h a 2 \ln h m h a+\pi \mu s$ U02um\{ßhs+lnhmhs $h m 2-h a 2-2 h m 2 \ln h m h a(1+A)$

And 


$$
\begin{aligned}
& \begin{array}{l}
Q_{s}=\frac{\pi \phi_{m_{0}}}{8 \mu_{m}}\left[\frac{2 \beta\left(h_{s}^{2}-h_{m}^{2}\right)-h_{s}\left\{\left(h_{s}^{2}-h_{m}^{2}\right)+2 h_{m}^{2} \ln \left(\frac{h_{m}}{h_{s}}\right)\right\}}{h_{s}\left\{\frac{\beta}{h_{s}}+\ln \left(\frac{h_{m}}{h_{s}}\right)\right\}}\right]\left\{2 h_{m}^{2} \ln \left(\frac{h_{m}}{h_{a}}\right)-\left(h_{m}^{2}-h_{a}^{2}\right)\right\}(1+A) \\
+\frac{\pi \phi_{s_{0}}}{8 \mu_{m}}\left[\frac{2 \beta\left(h_{s}^{2}-h_{m}^{2}\right)-h_{s}\left\{\left(h_{s}^{2}-h_{m}^{2}\right)+2 h_{m}^{2} \ln \left(\frac{h_{m}}{h_{s}}\right)\right\}}{h_{s}\left\{\frac{\beta}{h_{s}}+\ln \left(\frac{h_{m}}{h_{s}}\right)\right\}}\right]\left\{\frac{\left\{2 \beta h_{s}-\left(h_{s}^{2}-h_{m}^{2}\right)\right\}}{\left\{\frac{\beta}{h_{s}}+\ln \left(\frac{h_{m}}{h_{s}}\right)\right\}}-2 h_{m}^{2}\right\} \ln \left(\frac{h_{m}}{h_{a}}\right)(1+A) \\
+\frac{\pi \phi_{s_{0}}}{8 \mu_{s}}\left[\frac{\left(h_{s}^{2}-h_{m}^{2}-2 \beta h_{s}\right)\left(h_{s}^{2}-h_{m}^{2}+2 h_{s}^{2} \ln \left(\frac{h_{m}}{h_{s}}\right)\right.}{\left\{\frac{\beta}{h_{s}}+\ln \left(\frac{h_{m}}{h_{s}}\right)\right\}}-\left(h_{s}^{2}-h_{m}^{2}\right)^{2}\right] \\
+\frac{\pi U_{a}}{2}\left[\frac{\left.2 \beta\left(h_{s}^{2}-h_{m}^{2}\right)-h_{s}\left\{\left(h_{s}^{2}-h_{m}^{2}\right)\right\}+2 h_{m}^{2} \ln \left(\frac{h_{m}}{h_{s}}\right)\right\}}{h_{s}\left\{\frac{\beta}{h_{s}}+\ln \left(\frac{h_{m}}{h_{s}}\right)\right\}}\right](1+A)+\frac{\pi U_{0}}{2}\left\{\frac{\left(h_{s}^{2}-h_{m}^{2}\right)+2 h_{s}^{2} \ln \left(\frac{h_{m}}{h_{s}}\right)}{\left\{\frac{\beta}{h_{s}}+\ln \left(\frac{h_{m}}{h_{s}}\right)\right\}}\right\} \\
+\frac{\pi U_{0}}{2}\left[\frac{h_{s}\left\{\left(h_{s}^{2}-h_{m}^{2}\right)+2 h_{m}^{2} \ln \left(\frac{h_{m}}{h_{s}}\right)\right\}-2 \beta\left(h_{s}^{2}-h_{m}^{2}\right)}{h_{s}\left\{\frac{\beta}{h_{s}}+\ln \left(\frac{h_{m}}{h_{s}}\right)\right\}}\right] A(1+A)(\mathbf{1 9 )}
\end{array} \\
& \text { where } A=\left[\frac{\mu_{s} \ln \left(\frac{h_{m}}{h_{a}}\right)}{\mu_{m}\left\{\frac{\beta}{h_{s}}+\ln \left(\frac{h_{m}}{h_{s}}\right)\right\}}\right]
\end{aligned}
$$

\section{Results and Discussion}

To study the effect of various parameters on mucus transport rate quantitatively, the expression for $Q_{m}$ can be written in non- dimensional form as:

$$
\begin{gathered}
\bar{Q}_{m}= \\
\frac{\pi \bar{\phi}_{m_{0}}}{8 \bar{\mu}_{m}}\left[\left(\bar{h}_{m}^{4}-\bar{h}_{a}^{4}\right)-\frac{\left(\bar{h}_{m}^{2}-\bar{h}_{a}^{2}\right)^{2}}{\ln \left(\frac{\bar{h}_{m}}{\bar{h}_{a}}\right)}\right]+\frac{\pi \bar{\phi}_{m_{0}}}{8 \bar{\mu}_{m} \ln \left(\frac{\bar{h}_{m}}{\bar{h}_{a}}\right)}\left[2 \bar{h}_{m}^{2} \ln \left(\frac{\bar{h}_{m}}{\bar{h}_{a}}\right)-\right. \\
h m 2-h a 22(1+A)+\pi \phi s 08 \mu m 2 \beta+h m^{2}-1 \beta+\ln h m-2 h m
\end{gathered}
$$
$h m 2-h a 22(1+A)+\pi \phi s 08 \mu m 2 \beta+h m 2-1 \beta+\ln h m-2 h m$ $22 \mathrm{hm} 2 \ln h m h a-h m 2-h a 2(1+A)+\pi U a 2 \ln h m h a 2 h m 2 \mathrm{ln}$

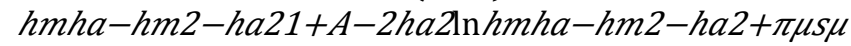
$m \beta+\ln h m 12 h m 2-h a 2-h m 21 n h m h a$

where $\bar{A}=\frac{\bar{\mu}_{s} \ln \left(\frac{\bar{h}_{m}}{\bar{h}_{a}}\right)}{\bar{\mu}_{m}\left(\bar{\beta}+\ln \bar{h}_{m}\right)}$

by using the following non-dimensional parameters:

$$
\begin{gathered}
\bar{\beta}=\frac{\beta}{h_{s}}, \bar{h}_{m}=\frac{h_{m}}{h_{s}}, \bar{h}_{a}=\frac{h_{a}}{h_{s}}, \overline{\mu_{s}}=\frac{\mu_{s}}{\mu_{0}}, \overline{\mu_{m}}=\frac{\mu_{m}}{\mu_{0}} \\
\bar{U}_{a}=\frac{U_{a}}{U_{0}}, \bar{\phi}_{s_{0}}=\frac{\phi_{s_{0}} h_{s}^{2}}{\mu_{0} U_{0}}, \bar{\phi}_{m_{0}}=\frac{\phi_{m_{0}} h_{s}^{2}}{\mu_{0} U_{0}}, \bar{Q}_{m}=\frac{Q_{m}}{U_{0} h_{s}^{2}}
\end{gathered}
$$

where $\mu_{0}$ is the viscosity of the serous sub-layer fluid in contact with epithelium. $\bar{Q}_{m}$ given by (20) is plotted in Fig. 2-5 using the following set of parameters which have been calculated by using typical values of various characteristics related to airways [King et al.(1993)]:

$\bar{\beta}=0.01-0.04, \bar{h}_{m}=0.996-0.998, \bar{h}_{a}=0.952$, $\overline{\mu_{s}}=1-4$,

$\overline{\mu_{m}}=10-100, \bar{U}_{a}=1.0-1.5, \bar{\phi}_{s_{0}}=5, \bar{\phi}_{m_{0}}=1-10$

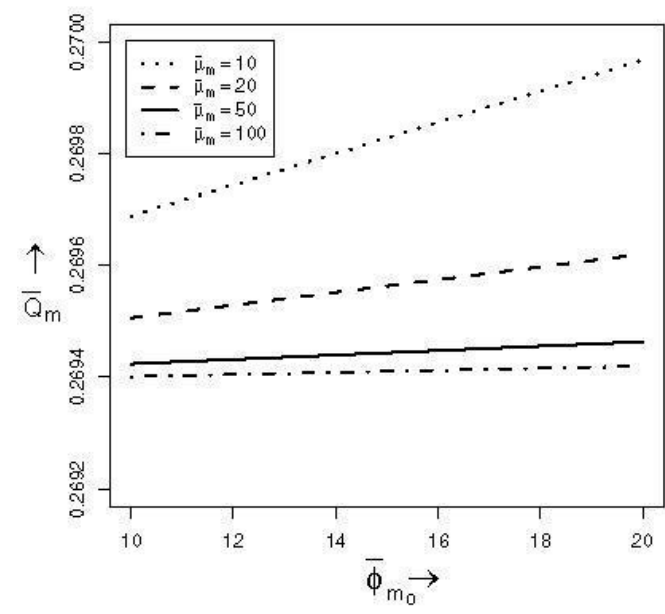

Figure 2: Variation of $\bar{Q}_{m}$ with $\bar{\phi}_{m_{0}}$ for different values of $\bar{\mu}_{m}$

Fig.2 shows the variation of $\bar{Q}_{m}$ with $\bar{\phi}_{m_{0}}$ for different values of $\bar{\mu}_{m}$ and fixed values of $\bar{\beta}=0.01, \bar{h}_{a}=0.952$, $\bar{h}_{m}=0.996, \bar{\mu}_{s}=1.0, \bar{U}_{a}=1.0$ and $\bar{\phi}_{s_{0}}=5$. Here, it is seen that the mucus transport rate increases as the pressure drop or gravitational force due to gravity increases, but it decreases with increase in mucus viscosity, the relative decrease being larger at higher values of the pressure drop or gravitational force. These results are in line with the conclusion drawn by King et al. (1993) and Agarwal and Verma (1997) in their mathematical models. 


\section{International Journal of Science and Research (IJSR)

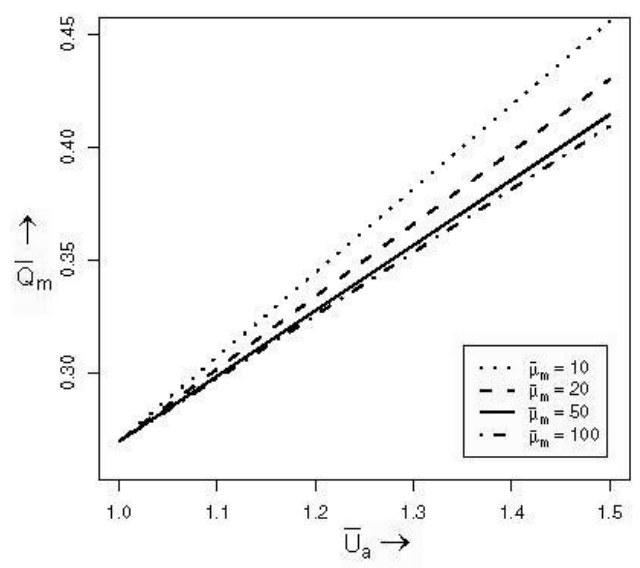

Figure 3: Variation of $\bar{Q}_{m}$ with $\bar{U}_{a}$ for different values of $\bar{\mu}_{m}$

Fig.3 shows the variation of $\bar{Q}_{m}$ with $\bar{U}_{a}$ for different values of $\bar{\mu}_{m}$ and fixed values of $\bar{\beta}=0.01, \bar{h}_{a}=0.952, \bar{h}_{m}=$ $0.996, \bar{\mu}_{s}=1.0, \bar{\phi}_{s_{0}}=5$ and $\bar{\phi}_{m_{0}}=10$. This figure illustrates that the mucus transport rate increases as the airvelocity generated by air-motion at the mucus air interface increases, but it decreases as its viscosity increases.

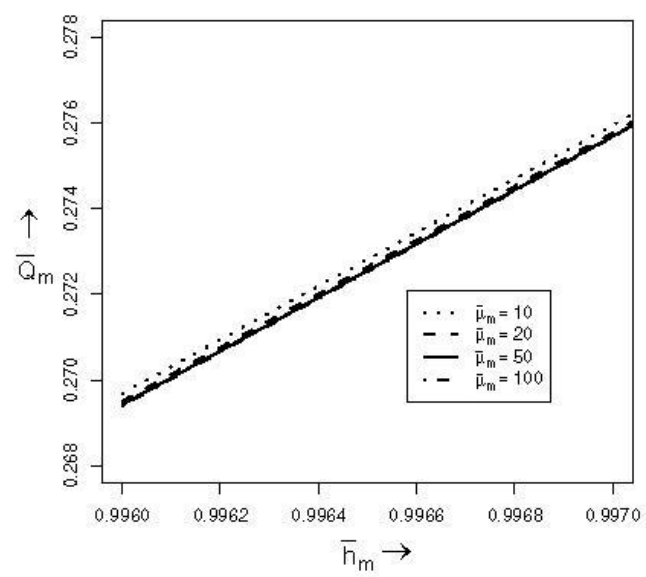

Figure 4: Variation of $\bar{Q}_{m}$ with $\bar{h}_{m}$ for different values of $\bar{\mu}_{m}$

Fig.4 shows the variation of $\bar{Q}_{m}$ with $\bar{h}_{m}$ for different values of $\bar{\mu}_{m}$ and fixed values of $\bar{\beta}=0.01, \bar{h}_{a}=0.952$ $, \bar{h}_{m}=0.996, \bar{\mu}_{s}=1.0, \bar{U}_{a}=1.0, \bar{\phi}_{s_{0}}=5$ and $\bar{\phi}_{m_{0}}=$ 10. This figure illustrates that mucus flow rate increases as the mucus layer thickness increases. It is also seen that mucus transport rate decreases as the viscosity of mucus layer increases.

Fig.5 shows the variation of $\bar{Q}_{m}$ with $\bar{\beta}$ for different values of $\bar{\mu}_{m}$ and for fixed vales of $\bar{h}_{a}=0.952, \bar{h}_{m}=0.996$ $, \bar{\mu}_{s}=1.0, \bar{U}_{a}=1.0, \bar{\phi}_{s_{0}}=5$ and $\bar{\phi}_{m_{0}}=10$. This figure illustrates that the mucus flow rate increases as the porosity parameter increases, but it decreases with increase in its viscosity.

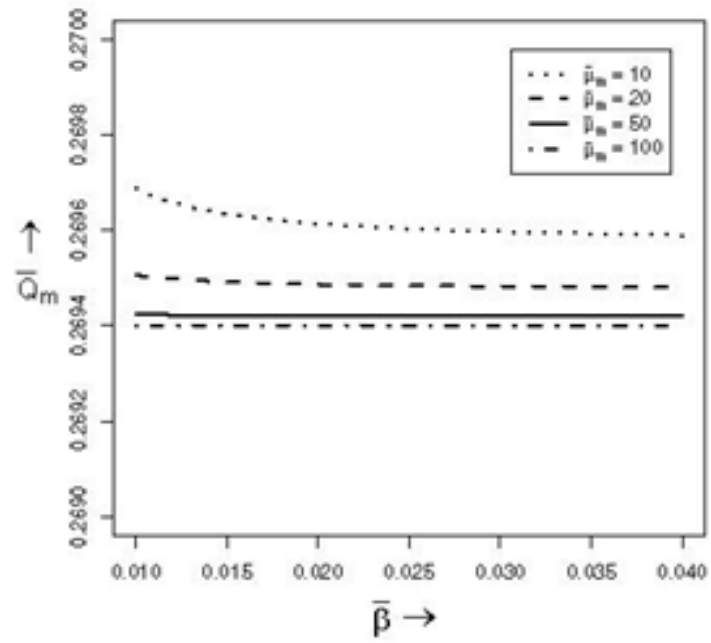

Figure 5: Variation of $\bar{Q}_{m}$ with $\bar{\beta}$ for different values of $\bar{\mu}_{m}$

These observations are in line with the conclusions drawn by Agarwal and Verma (1997) and Verma (2010) in their studies.

\section{Conclusion}

This study concludes that the mucus flow rate increases with increase in air velocity due to air flow, cilia tip velocity due to beating cilia, porosity parameter due to immotile cilia, mucus thickness, pressure drop in the fluid layers as well as gravitational force due to gravity. It also concludes that the mucus transport rate decreases with increase in mucus viscosity.

\section{References}

[1] Agarwal, M. and Verma, V. S., "A planar model for muco-ciliary transport: Effect of air motion and porosity", Proc. Nat. Acad. Sci. India, 67 (A) II, (1997), pp. 193-204.

[2] Barton, C. and Raynor, S., "Analytical investigation of cilia induced mucus flow”, Bull. Math. Biophys. 29, (1967), pp.419-428.

[3] Beavers, J. S. and Joseph, D. D., Boundry conditions at a naturally permeable wall, J. Fluid Mech., 30 (1), (1967), pp.197-207.

[4] Blake, J.R, "On the movement of mucus in the lung", J. Biomech.8, (1975), pp.179-190.

[5] Blake, J. R. and Winet, H., "On the mechanics of muco-ciliary transport”, Biorheology,17, (1980), pp.125-134.

[6] King, M., Agarwal, M. and Shukla , J., B., "A planar model for muco-ciliary transport : Effect of mucus visco-elasticity", Biorheology ,30, (1993), pp.49-61.

[7] King, M.,Brock, G. and A Lundell, C., "Clearance of mucus by simulated cough", J. Appl._Physiol. , 58, (1985), pp.11776-1782.

[8] King , M., Jahm, J. M., Pierrot, D., Vaquez-Girod, S. and Puchelle, E., "The role mucus gel viscosity, spinnability and adhesive property in clearance by simulated cough", Biorheology, 26, (1989), pp.737-745.

[9] Ross, S.M. andCorrsin, S., "Results of an analytical of muco-ciliary pumping”, J. Appl. Physiol., 37, 3(1974), pp.33-340. 


\section{International Journal of Science and Research (IJSR) \\ ISSN (Online): 2319-7064}

Index Copernicus Value (2013): 6.14 | Impact Factor (2014): 5.611

[10] Sieigh, M. A.,Blake , J. R. and Liron, N., "The propulsion of mucus by cilia”, Am. Rev._Respir. Dis., 137, (1988), pp.726-741.

[11] Verma, V. S., "A mathematical study on mucus transport in the lung", J. Nat. Acad. Math., 21, (2007),pp.107-117.

[12] Vema, V. S., “A planar for mucus transport in human respiratory tract : Effects air flow, porosity and mucus visco-elastity” ,J. Nat. Acad. Math., (2010), 24,53-60.

[13] Verma, V. S. and Tripathee, S.M. (2011): A study on mucus flow in human lung airways, Journal of Progressive Science, 02(01), 113-120.

[14] Verma, V.S. and Tripathee, S.M. (2013): A planar model for muco-ciliary transport in the human lung: Effects of mucus visco-elasticity, cilia beating and porosity, IJMRS's Int J. Mathematical Modelling and Physical Sciences, 01 (01); 19-25. 